\title{
CAPACITY OF SETS AND FOURIER SERIES
}

BY

\author{
R. SALEM AND A. ZYGMUND
}

Introduction. In $1939 \mathrm{~A}$. Beurling [1](1) proved that if the series $\sum n\left(a_{n}{ }^{2}+b_{n}{ }^{2}\right)$ converges then the points of divergence of the Fourier series $a_{0} / 2+\sum_{1}^{\infty} a_{n} \cos n x+b_{n} \sin n x$ form a set of logarithmic capacity zero. In the same paper, he stated the following result, the proof of which was due to appear, but to our knowledge has not appeared, in the Arkiv för Matematik: if $0<\alpha \leqq 1$ and if the series $\sum n^{\alpha-\epsilon}\left(a_{n}^{2}+b_{n}{ }^{2}\right)$ converges for every $\epsilon>0$, the above Fourier series is summable by Abel's method except in a set whose $\beta$-capacity is zero for every $\beta>1-\alpha$.

The present paper has its origin in the desire of the authors to find a more direct and simpler proof of Beurling's results and to extend them. In the course of the work, however, some other theorems of independent interest have been established, and are presented here.

The paper is divided into four parts. In the first we give in terms of Fourier series a necessary and sufficient condition for a linear set $E$ to be of positive $\alpha$-capacity.

The second is devoted to the proof of the following theorem which includes the results of Beurling already quoted: If $0<\alpha \leqq 1$ and if the series $\sum n^{\alpha}\left(a_{n}^{2}+b_{n}^{2}\right)$ converges, the set of points of divergence of the Fourier series $a_{0} / 2+\sum_{1}^{\infty} a_{n} \cos n x+b_{n} \sin n x$ is of $(1-\alpha)$-capacity zero if $\alpha \neq 1$ and of logarithmic capacity zero if $\alpha=1$.

In the third part we prove some theorems on equi-summability and equiconvergence of certain series and certain integrals of the Dini type.

In the fourth part we apply the preceding results to some theorems concerning the existence of certain integrals of the Dini type,

$$
\begin{aligned}
& \int_{0}^{\pi} \frac{f(x+t)-f(x-t)}{t^{1+\alpha}} d t, \quad \int_{0}^{\pi} \frac{f(x+t)+f(x-t)-2 f(x)}{t^{1+\alpha}} d t, \\
& 0<\alpha<1 ; \epsilon \rightarrow+0,
\end{aligned}
$$

and we apply them to some minor, and partly known, results on the capacity of linear sets.

It will be convenient, in order to avoid unnecessary repetitions, to recall here certain definitions and notations which will be used throughout the paper.

We shall consider in the plane either logarithmic potentials of the form

Presented to the Society, September 17, 1945; received by the editors May 9, 1945.

(1) Numbers in brackets refer to the Bibliography at the end of the paper. 
$\int d \mu / \rho$, or generalized potentials of the form $\int d \mu / \rho^{\alpha}(0<\alpha<1)$. The distribution shall always be supposed to be spread on the circumference of the unit circle $|z|=1, z=r e^{i x}$, and to be a non-negative additive function of sets, so that the point function $\mu(x)$ is a nondecreasing function for $0 \leqq x \leqq 2 \pi$. The total distribution $\mu(2 \pi)-\mu(0)$ will be supposed equal to 1 , unless otherwise stated. The sets considered will always be Borel sets.

We shall say that a distribution $\mu$ is "concentrated" on a set $E$ if $\int_{E} d \mu=\int_{0}^{2 \pi} d \mu$, in other words if the integral over the complementary set of $E$ is zero.

As is well known (see Frostman [2, p. 48], de la Vallée Poussin [3, p. 20]), the set $E$ is said to be of positive logarithmic capacity if there exists a positive distribution $\mu$ concentrated on $E$ such that the potential

$$
V=\int_{0}^{2 \pi} \log \frac{1}{\left|e^{i t}-r e^{i x}\right|} d \mu(t)
$$

is bounded uniformly in $x$ as $r \rightarrow 1$. In the same way, a set $E$ is said to be of positive $\alpha$-capacity $(0<\alpha<1)$ if there exists a positive distribution $\mu$ concentrated on $E$ and such that the generalized potential

$$
V=\int_{0}^{2 \pi} \frac{d \mu(t)}{\left|e^{i t}-r e^{i x}\right|^{\alpha}}
$$

is bounded uniformly in $x$ as $r \rightarrow 1$.

It is well known that there is a connection between positive $\alpha$-capacity and positive Hausdorff measure of order $\alpha$. If a set is of positive $\alpha$-capacity, its Hausdorff measure of order $\alpha$ is positive; on the other hand, if a closed set has a positive Hausdorff measure of order $\alpha$ its $(\alpha-\epsilon)$ capacity is positive for $\epsilon>0$. (See Frostman [2, p. 86)], and the various references quoted there.) It follows that if a set $E$ is of $\alpha$-capacity zero, any closed subset of $E$ has a Hausdorff dimension of order $\alpha+\epsilon$ equal to zero.

We shall consider continuous functions $f(x)$ of period $2 \pi(f(2 \pi)=f(0))$ satisfying a Lipschitz condition and we say that $f(x)$ belongs to Lip $\alpha$ $(0<\alpha \leqq 1)$ if

$$
|f(x+h)-f(x)|^{\circ}=O\left(|h|^{\alpha}\right)
$$

uniformly in $x$ as $h \rightarrow 0$. We shall say, following a notation already used (see Zygmund [7]), that $f$ belongs to lip $\alpha$ if $|f(x+h)-f(x)|=o(|h| \alpha)$.

Let $a_{0} / 2+\sum_{1}^{\infty}\left(a_{n} \cos n x+b_{n} \sin n x\right)$ be the Fourier series of a continuous function $f(x)$ of bounded variation and of period $2 \pi$. It is clear that such a function can not be monotonic in $(0,2 \pi)$ since $f(0)=f(2 \pi)$; but we shall say that $f(x)$ belongs to the monotonic type if the Fourier-Stieltjes series

$$
d(f+C x) \sim C+\sum_{1}^{\infty}\left(n b_{n} \cos n x-n a_{n} \sin n x\right)
$$


is, for some $C$, the Fourier-Stieltjes series of a monotonic function; in other words if there exists a constant $C$ such that $f(x)+C x$ is either never decreasing or never increasing in $(-\infty,+\infty)$.

Finally we shall have to quote various known results which can be found in Zygmund [8]. Hereafter we shall refer to this book by the initials T.S.

1. A necessary and sufficient condition for a set $E$ to be of positive $\alpha$-capacity.

THEOREM I. The necessary and sufficient condition that a set $E$ be of positive capacity with respect to the potential function $1 / r^{\alpha}(0<\alpha<1)$ is that a positive distribution $\mu$ exist concentrated on $E$ such that if the Fourier-Stieltjes series of $d \mu(x)$ is $d \mu \sim 1 / 2 \pi+\sum_{1}^{\infty} a_{n} \cos n x+b_{n} \sin n x$ the series $\sum_{1}^{\infty}\left(a_{n} \cos n x+b_{n} \sin n x\right) / n^{1-\alpha}$ is the Fourier series of a bounded function. The theorem holds for $\alpha=0$, the potential being in this case the logarithmic potential.

Proof. Suppose first that $0<\alpha<1$ and suppose that the $\alpha$-capacity of $E$ is positive. Then there exists a positive distribution $\mu$,

$$
d \mu \sim \frac{1}{2 \pi}+\sum_{1}^{\infty} a_{n} \cos n x+b_{n} \sin n x,
$$

concentrated on $E$ and such that the potential

$$
V=\int_{0}^{2 \pi} \frac{d \mu(t)}{\left|e^{i t}-r e^{i x}\right|^{\alpha}}
$$

is bounded uniformly in $x$, as $r \rightarrow 1$. Hence the integral

$$
U=\int_{0}^{2 \pi} \frac{d \mu(t)}{\left[1-r e^{i(x-t)}\right]^{\alpha}}
$$

is also uniformly bounded. If $r<1$, we have

$$
\begin{aligned}
{\left[1-r e^{i(x-t)}\right]^{-\alpha}=1 } & +\frac{\alpha}{1} r e^{i(x-t)}+\cdots \\
& +\frac{\alpha(\alpha+1) \cdots(\alpha+n-1)}{n !} r^{n} e^{n i(x-t)}+\cdots
\end{aligned}
$$

Thus, considering only the real part of $U$, we see that the function

where

$$
\sum_{1}^{\infty} r^{n}\left(a_{n} \cos n x+b_{n} \sin n x\right) \gamma_{n}
$$

$$
\gamma_{n}=\frac{\alpha(\alpha+1) \cdots(\alpha+n-1)}{n !},
$$

is uniformly bounded as $r \rightarrow 1$. Now it is well known that 


$$
\gamma_{n}=\frac{1}{\Gamma(\alpha)} \frac{1}{n^{1-\alpha}}\left[1+o\left(\frac{1}{n}\right)\right] \text {. }
$$

Hence the function

$$
\sum_{1}^{\infty} \frac{a_{n} \cos n x+b_{n} \sin n x}{n^{1-\alpha}} r^{n}
$$

is uniformly bounded as $r \rightarrow 1$, which proves the necessity of our condition.

To prove that the condition is sufficient we observe first that if it is satisfied, then, by the preceding argument, the real part of $U$ is bounded as $r \rightarrow 1-0$ for a certain positive distribution $\mu$ concentrated on $E$.

Now, if $r<1$, we have for every argument $\phi$

$$
1-r e^{i \phi}=\left|1-r e^{i \phi}\right| e^{i \theta},
$$

the argument $\theta$ of $1-r e^{i \phi}$ being included between $-\pi / 2$ and $+\pi / 2$. Hence

$$
R\left(1-r e^{i \phi}\right)^{-\alpha}=\left|1-r e^{i \phi}\right|^{-\alpha} \cos \alpha \theta,
$$

where $\cos \alpha \theta$ is included between the two positive quantities $\cos \pi \alpha / 2$ and 1 . It follows immediately that the boundedness of the real part of $U$ implies the boundedness of $V$, which proves that the set is of positive $\alpha$-capacity. Incidentally we have proved that the boundedness of $\sum\left(\left(a_{n} \cos n x+b_{n} \sin n x\right) / n^{1-\alpha}\right) r^{n}$ implies the boundedness of the conjugate series $\sum\left(\left(a_{n} \sin n x-b_{n} \cos n x\right) / n^{1-\alpha}\right) r^{n}$, since the boundedness of $V$ involves the boundedness of the imaginary part of $U$. This fact will be useful later.

The case $\alpha=0$ is much simpler, since for the logarithmic potential we have the harmonic function

$$
\begin{aligned}
\int_{0}^{2 \pi} \log \frac{1}{\left|e^{i t}-r e^{i x}\right|} d \mu(t) & =\int_{0}^{2 \pi} \frac{1}{2} \log \frac{1}{1-2 r \cos (x-t)+r^{2}} d \mu(t) \\
& =\int_{0}^{2 \pi}\left(\sum_{1}^{\infty} \frac{\cos n(x-t)}{n} r^{n}\right) d \mu(t) \\
& =\pi \sum_{1}^{\infty} \frac{a_{n} \cos n x+b_{n} \sin n x}{n} r^{n} .
\end{aligned}
$$

This completes the proof of the theorem.

Corollary. If the set $E$ is of positive $\alpha$-capacity $(0<\alpha<1)$ it is possible to find a positive distribution $\mu$, concentrated on $E$, such that the function $\mu(x)$ belongs to $\operatorname{Lip} \alpha$.

For, by the argument of the preceding theorem and considering also the imaginary part of $U$, we see that both the series $S=\sum_{1}^{\infty}\left(a_{n} \cos n x+b_{n} \sin n x\right) / n^{1-\alpha}$ and its conjugate $T$ are Fourier series of bounded functions; hence, by a well 
known theorem (see T.S. p. 225), the fractional integral of order $\alpha$ of $S+i T$ belongs to Lip $\alpha$. In particular, the function

$$
\sum_{1}^{\infty} \frac{a_{n} \sin n x-b_{n} \cos n x}{n}
$$

representing $\mu(x)-x / 2 \pi+$ const. belongs to $\operatorname{Lip} \alpha$, which proves our corollary.

It will be shown later on that the existence of a positive distribution $\mu$, concentrated on $E$ and such that $\mu(x)$ belongs to $\operatorname{Lip} \alpha$, is not sufficient for the set $E$ to be of positive $\alpha$-capacity.

2. Theorems on the convergence of Fourier series.

THEOREM II. Let $a_{0} / 2+\sum_{1}^{\infty}\left(a_{n} \cos n x+b_{n} \sin n x\right)$ be a Fourier series $(S)$ such that $\sum_{1}^{\infty} n^{\alpha}\left(a_{n}{ }^{2}+b_{n}{ }^{2}\right)<\infty, 0<\alpha<1$. Then the set $E$ of the points of divergence of $S$ is of $(1-\alpha)$-capacity zero. The theorem holds for $\alpha=1$, it being understood that in this case the logarithmic capacity of $E$ is zero.

The proof will be based on the following lemma.

LEMmA. Let $H(x)=\sum_{1}^{\infty} \cos k x / k^{\beta}, 0<\beta<1$, and $H_{n}(x)=\sum_{1}^{n} \cos k x / k^{\beta}$. Then $\left|H_{n}(x)\right|<A H(x)+B, A$ and $B$ being positive constants depending on $\beta$ only. (If $x=0$, the second member of the inequality is to be interpreted as $+\infty$.)

Suppose, as we may, that $0<x \leqq \pi$. We observe first tha+ if $n \leqq 1 / x$, we have

$$
\left|H_{n}(x)\right| \leqq \sum_{1}^{n} \frac{1}{k^{\beta}}=O\left(n^{1-\beta}\right)=O\left(x^{\beta-1}\right) .
$$

Supposing now that $n>1 / x$, we have

$$
H_{n}(x)=\sum_{1}^{[1 / x]} \frac{\cos k x}{k^{\beta}}+\sum_{[1 / x]}^{n} \frac{\cos k x}{k^{\beta}}=S_{1}+S_{2},
$$

say. Now, as before, $S_{1}=O\left(x^{\beta-1}\right)$. On the other hand, if we apply Abel's transformation,

$$
S_{2}=\sum_{[1 / x]}^{n-1}\left[\frac{1}{k^{\beta}}-\frac{1}{(k+1)^{\beta}}\right] T_{k}(x)+\frac{1}{n^{\beta}} T_{n}(x),
$$

where $T_{k}(x)=\sum_{[1 / x]}^{k} \cos \nu x=O(1 / x)$. Hence

$$
\left|S_{2}\right|<x^{\beta} O\left(\frac{1}{x}\right)+\frac{1}{n^{\beta}} O\left(\frac{1}{x}\right)=O\left(x^{\beta-1}\right),
$$

and thus we see that for $0 \leqq x \leqq \pi$

$$
\left|H_{n}(x)\right|<C x^{\beta-1},
$$

$C$ depending on $\beta$ only. But it is well known that $1 / 2+H(x)$ is a positive func- 
tion, and that as $x \rightarrow 0$ it is asymptotically equal to $|x|^{\beta-1} \sin (\pi \beta / 2) \Gamma(1-\beta)$ (see T.S. pp. 109 and 129). Hence for all $x$ we may write $\left|H_{n}(x)\right|<A H(x)$ $+B, A$ and $B$ being positive constants depending on $\beta$ only, which proves our lemma.

Now, to prove our theorem let us suppose first that $0<\alpha<1$, and let us assume that $E$ is of positive $(1-\alpha)$-capacity. Then by Theorem I we can find a positive distribution $\mu$, concentrated on $E$, such that, if $d \mu \sim 1 / 2 \pi$ $+\sum_{1}^{\infty} \alpha_{n} \cos n x+\beta_{n} \sin n x$, the series $\sum\left(\alpha_{n} \cos n x+\beta_{n} \sin n x\right) / n^{\alpha}$ is the Fourier series of a bounded function.

Let $\omega(n)$ be a positive monotonic function increasing infinitely with $n$ and such that $\sum n^{\alpha} \omega(n)\left(a_{n}^{2}+b_{n}{ }^{2}\right)<\infty$. Let $A_{n}=a_{n}(\omega(n))^{1 / 2} B_{n}=b_{n}(\omega(n))^{1 / 2}$. Then in the set $E$ the partial sums $S_{n}(x)$ of the series $\sum_{1}^{\infty} A_{n} \cos n x+B_{n} \sin n x$ are unbounded (for their boundedness at a point $x$ would imply the convergence of $(S)$ at $x)$. Thus, if $n(x)$ denotes a positive integer and measurable function of $x$, with $n(x) \leqq n$, the integral $\int_{0}^{2 \pi} S_{n(x)}(x) d \mu$ can be made to increase infinitely in absolute value with $n$, for a suitable choice of $n(x)$ for each $n$. (The argument is an adaptation of a well known idea due to Kolmogoroff-Seliverstoff and Plessner; see T.S. p. 253. The difference is that here we use a Stieltjes integral instead of the integral $\int_{0}^{2 \pi} S_{n(x)}(x) d x$.)

We shall show that $\int_{0}^{2 \pi} S_{n(x)}(x) d \mu$ is bounded, thus getting a contradiction which will prove our theorem.

In fact, since $\sum n^{\alpha}\left(A_{n}{ }^{2}+B_{n}{ }^{2}\right)<\infty$, the series $\sum_{1}^{\infty}\left(A_{n} \cos n x+B_{n} \sin n x\right) n^{\alpha / 2}$ is the Fourier series of a function $F \in L^{2}$. We have

$$
A_{k} \cos k x+B_{k} \sin k x=\frac{1}{\pi} \int_{0}^{2 \pi} \frac{F(t) \cos k(t-x)}{k^{\alpha / 2}} d t .
$$

Hence, putting $G_{n}(x)=\sum_{1}^{n} \cos k x / k^{\alpha / 2}$, we have

$$
S_{n(x)}(x)=\sum_{1}^{n(x)} A_{k} \cos k x+B_{k} \sin k x=\frac{1}{\pi} \int_{0}^{2 \pi} F(t) G_{n(x)}(t-x) d t .
$$

Thus

$$
I=\int_{0}^{2 \pi} S_{n(x)}(x) d \mu(x)=\frac{1}{\pi} \int_{0}^{2 \pi} F(t) d t \int_{0}^{2 \pi} G_{n(x)}(t-x) d \mu(x),
$$

and so, by Schwarz's inequality,

$$
\pi^{2} I^{2}<\int_{0}^{2 \pi} F^{2}(t) d t \int_{0}^{2 \pi} d t \int_{0}^{2 \pi} \int_{0}^{2 \pi} G_{n(x)}(t-x) G_{n(y)}(t-y) d \mu(x) d \mu(y) .
$$

Now

$$
\int_{0}^{2 \pi} G_{n(x)}(t-x) G_{n(y)}(t-y) d t=\pi H_{n(x, y)}(x-y),
$$


where $H_{n}(x)=\sum_{1}^{n} \cos k x / k^{\alpha}$ and $n(x, y)=\min [n(x), n(y)]$. Thus, putting $A=\int_{0}^{2 \pi} F^{2}(t) d t$, we have

$$
\begin{aligned}
\pi I^{2} & <A \int_{0}^{2 \pi} \int_{0}^{2 \pi}\left|H_{n(x, y)}(x-y)\right| d \mu(x) d \mu(y) \\
& <A \int_{0}^{2 \pi} \int_{0}^{2 \pi}\left\{\left|H_{n(x)}(x-y)\right|+\left|H_{n(y)}(x-y)\right|\right\} d \mu(x) d \mu(y) \\
& =2 A \int_{0}^{2 \pi} \int_{0}^{2 \pi}\left|H_{n(y)}(x-y)\right| d \mu(x) d \mu(y) \\
& =2 A \int_{0}^{2 \pi} d \mu(y) \int_{0}^{2 \pi}\left|H_{n(y)}(x-y)\right| d \mu(x) .
\end{aligned}
$$

Now, using the lemma proved above, and putting $H(x)=\sum_{1}^{\infty} \cos k x / k^{\alpha}$, we have

$$
\int_{0}^{2 \pi}\left|H_{m}(x-y)\right| d \mu(x) \leqq A_{1} \int_{0}^{2 \pi} H(x-y) d \mu(x)+B,
$$

$A_{1}$ and $B$ being positive constants. By a well known theorem, the Fourier series of $\pi^{-1} \int_{0}^{2 \pi} H(x-y) d \mu(x)$ is $\sum_{1}^{\infty}\left(\alpha_{n} \cos n y+\beta_{n} \sin n y\right) / n^{\alpha}$, which by our assumption is the Fourier series of a bounded function. Hence, for almost all $y$,

$$
\frac{1}{\pi} \int_{0}^{2 \pi} H(x-y) d \mu(x)<M
$$

$M$ being a constant. Thus, for almost all $y$,

$$
\int_{0}^{2 \pi}\left|H_{m}(x-y)\right| d \mu(x) \leqq A_{1} M \pi+B .
$$

But the left-hand side here is continuous in $y$. Hence this inequality holds for all $y$, which proves the boundedness of the integral $I$, and hence our theorem.

The proof for the case $\alpha=1$ follows exactly the same line, but is simpler, since we do not need to use the lemma. We observe only that the coefficients of $H(x)=\sum_{1}^{\infty} \cos k x / k$ are $O(1 / k)$, and since $H(x)>-1 / 2, H_{n}(x)$ is uniformly bounded below, so that it suffices to prove that

$$
\int_{0}^{2 \pi} H_{m}(x-y) d \mu(x)=\pi \sum_{1}^{m}\left(\alpha_{n} \cos n y+\beta_{n} \sin n y\right) / n
$$

is bounded. But this is a partial sum of the Fourier series of a function assumed to be bounded, and with coefficients $O(1 / n)$. Hence the result follows.

3. Theorems on equi-summability and equi-convergence of certain series and integrals. 
THEOREM III. Let $a_{0} / 2+\sum_{1}^{\infty}\left(a_{n} \cos n x+b_{n} \sin n x\right)$ be the Fourier series of $a$ continuous function $f(x)$ of period $2 \pi$, and belonging to lip $\alpha$, where $0<\alpha<1$. Then the difference

$$
\begin{aligned}
-\frac{1}{\pi} \Gamma(\alpha+1) \cos & \frac{\pi \alpha}{2} \int_{1-r}^{\infty} \frac{f(x+t)-f(x-t)}{t^{1+\alpha}} d t \\
& -\sum_{1}^{\infty}\left(a_{n} \sin n x-b_{n} \cos n x\right) n^{\alpha} r^{n}
\end{aligned}
$$

tends to zero uniformly in $x$, as $r \rightarrow 1-0$.

If $f$ belongs to $\operatorname{Lip} \alpha$, the above difference is bounded, uniformly in $x$.

The reader will notice the analogy between this theorem and the classical theorem on the equi-summability of the conjugate series of a Fourier series and of the corresponding integral (see T.S. p. 54).

We need the following two lemmas: the first one is an obvious adaptation of the well known theorem of S. Bernstein on the order of approximation by Fejér's polynomials; the second one is due to Hardy and Littlewood. Both proofs being very short, we give them here for the sake of completeness.

Lemma. 1. If $g(x)$ is of period $2 \pi$ and belongs to $\operatorname{Lip} \alpha(0<\alpha<1)$, $g(x) \sim \alpha_{0} / 2+\sum \alpha_{n} \cos n x+\beta_{n} \sin n x$, and $g(r, x)$ denotes the corresponding harmonic function $g(r, x)=\alpha_{0} / 2+\sum\left(\alpha_{n} \cos n x+\beta_{n} \sin n x\right) r^{n}$, then $g(r, x)-g(x)$ $=O(1-r)^{\alpha}$ as $r \rightarrow 1-0$, uniformly in $x$.

Proof. Denote, as usual, by $P_{r}(t)$ Poisson's kernel $\left(1-r^{2}\right) / 2\left(1-2 r \cos t+r^{2}\right)$. Since $P_{r}(t)<1 /(1-r)$ and $P_{r}(t)<(1-r) / 4 r \sin ^{2} t / 2$, we have

$$
\begin{aligned}
\pi|g(r, x)-g(x)| & =\left|\int_{0}^{\pi}[g(x+t)+g(x-t)-2 g(x)] P_{r}(t) d t\right| \\
& \leqq \frac{1}{1-r} \int_{0}^{1-r} O\left(t^{\alpha}\right) d t+(1-r) \int_{1-r}^{\pi} O\left(t^{\alpha-2}\right) d t \\
& =O(1-r)^{\alpha} .
\end{aligned}
$$

Obviously, if $g(x)$ belongs to lip $\alpha$, then $g(r, x)-g(x)=o(1-r)^{\alpha}$.

LEMma 2. Let $g(x)$ and $g(r, x)$ have the same meaning as in Lemma 1. Then $\partial g(r, x) / \partial x=O(1-r)^{\alpha-1}$.

Proof. We have

$$
\frac{\partial g(r, x)}{\partial x}=-\frac{1}{\pi} \int_{0}^{\pi}[g(x+t)-g(x-t)] P_{r}^{\prime}(t) d t
$$

where $P !(t)$ denotes the derivative of Poisson's kernel with respect to $t$. We have $P_{r}^{\prime}(t)=-\left(1-r^{2}\right) r \sin t /\left(1-2 r \cos t+r^{2}\right)^{2}$ and thus 
Hence

$$
\left|P_{r}^{\prime}(t)\right|<\frac{2 t}{(1-r)^{8}}, \quad\left|P_{r}^{\prime}(t)\right|<\frac{2(1-r) t}{\left(4 r \sin ^{2} t / 2\right)^{2}} .
$$

$$
\left|\frac{\partial g(r, x)}{\partial x}\right| \leqq \frac{1}{(1-r)^{3}} \int_{0}^{1-r} O\left(t^{1+\alpha}\right) d t+(1-r) \int_{1-r}^{\pi} O\left(t^{\alpha-3}\right) d t=O(1-r)^{\alpha-1}
$$

Here again, if $g(x)$ belongs to lip $\alpha$, then $\partial g(r, x) / \partial x=o(1-r)^{\alpha-1}$.

We can now prove our theorern. Let

$$
g(t)=f(x+t)-f(x-t) \sim-\sum_{1}^{\infty} 2 \sin n t\left[a_{n} \sin n x-b_{n} \cos n x\right]
$$

and

$$
g(r, t)=-\sum_{1}^{\infty} 2 \sin n t\left[a_{n} \sin n x-b_{n} \cos n x\right] r^{n} .
$$

For, given $x$ and $r(r<1)$, the series

$$
\frac{g(r, t)}{t^{1+\alpha}}=-2 \sum_{1}^{\infty} \frac{\sin n t}{t^{1+\alpha}}\left[a_{n} \sin n x-b_{n} \cos n x\right] r^{n}
$$

is uniformly convergent in $t$ for $t>\epsilon>0$. Hence we can integrate term by term in the interval $(\epsilon, T)$. Observing that

$$
\left|\int_{0}^{\epsilon} \frac{\sin n t}{t^{1+\alpha}} d t\right|<C n \epsilon^{1-\alpha}, \quad\left|\int_{T}^{\infty} \frac{\sin n t}{t^{1+\alpha}} d t\right|<\frac{C}{T^{\alpha}},
$$

$C$ depending on $\alpha$ only, we deduce immediately that

$$
\int_{0}^{\infty} \frac{g(r, t)}{t^{1+\alpha}} d t=-2 \sum_{1}^{\infty}\left(a_{n} \sin n x-b_{n} \cos n x\right) r^{n} \int_{0}^{\infty} \frac{\sin n t}{t^{1+\alpha}} d t .
$$

Now

Hence

$$
\begin{aligned}
\int_{0}^{\infty} \frac{\sin n t}{t^{1+\alpha}} d t & =n^{\alpha} \int_{0}^{\infty} \frac{\sin x}{x^{1+\alpha}} d x=\frac{n^{\alpha}}{\alpha} \int_{0}^{\infty} \frac{\cos x}{x^{\alpha}} d x \\
& =\frac{n^{\alpha}}{\alpha} \sin \frac{\pi \alpha}{2} \Gamma(1-\alpha)=\frac{n^{\alpha} \pi}{2 \cos (\pi \alpha / 2) \Gamma(\alpha+1)} .
\end{aligned}
$$

$$
-\frac{1}{\pi} \cos \frac{\pi \alpha}{2} \Gamma(\alpha+1) \int_{0}^{\infty} \frac{g(r, t)}{t^{1+\alpha}} d t=\sum_{1}^{\infty}\left(a_{n} \sin n x-b_{n} \cos n x\right) n^{\alpha} r^{n}
$$

and to prove our theorem it is sufficient to show that

$$
D=\int_{0}^{\infty} \frac{g(r, t)}{t^{1+\alpha}} d t-\int_{1-r}^{\infty} \frac{g(t)}{t^{1+\alpha}} d t
$$


is $o(1)$ if $g \in \operatorname{lip} \alpha$, and $O(1)$ if $g \in \operatorname{Lip} \alpha$, as $r \rightarrow 1-0$, uniformly in $x$. Suppose $g \in \operatorname{Lip} \alpha$ (the proof is identical in the other case). Write

$$
D=\int_{1-r}^{\infty} \frac{g(r, t)-g(t)}{t^{1+\alpha}} d t+\int_{0}^{1-r} \frac{g(r, t)}{t^{1+\alpha}} d t .
$$

By Lemma 1, we have, uniformly in $x$ and $t$,

$$
|g(r, t)-g(t)|=O(1-r)^{\alpha},
$$

and thus the first integral is $O(1)$. For the second integral we note that, since $g(r, 0)=0$, we have

$$
g(r, t)=t\left(\frac{\partial g(r, t)}{\partial t}\right)_{t=0}
$$

and thus, by Lemma $2,|g(r, t)|=t O(1-r)^{\alpha-1}$ so that the second integral is

$$
O\left[(1-r)^{\alpha-1} \int_{0}^{1-r} \frac{d t}{t^{\alpha}}\right]=O(1) .
$$

This completes the proof of the theorem.

The following theorem is analogous to Theorem III and can be proved by an almost identical argument.

THEOREM IV. The function $f$ having the same meaning as in Theorem III, the difference

$$
\begin{array}{r}
-\frac{1}{\pi} \sin \frac{\pi \alpha}{2} \Gamma(\alpha+1) \int_{1-r}^{\infty} \frac{f(x+t)+f(x-t)-2 f(x)}{t^{1+\alpha}} d t \\
-\sum_{1}^{\infty}\left(a_{n} \cos n x+b_{n} \sin n x\right) r^{n} n^{\alpha}
\end{array}
$$

tends to zero uniformly in $x$ as $r \rightarrow 1-0$ if $f \in \operatorname{lip} \alpha$, and is uniformly bounded if $f \in \operatorname{Lip} \alpha$.

We write, $f(r, x)$ being the harmonic function corresponding to $f(x)$,

$$
\begin{aligned}
G(t) & =f(x+t)+f(x-t)-2 f(x), \\
G(r, t) & =f(r, x+t)+f(r, x-t)-2 f(r, x) \\
& =-4 \sum_{1}^{\infty} \sin ^{2}\left(\frac{n t}{2}\right)\left(a_{n} \cos n x+b_{n} \sin n x\right) r^{n},
\end{aligned}
$$

and we observe that

$$
\begin{aligned}
\int_{0}^{\infty} \frac{\sin ^{2}(n t / 2)}{t^{1+\alpha}} d t & =\left(\frac{n}{2}\right)^{\alpha} \int_{0}^{\infty} \frac{\sin ^{2} x}{x^{1+\alpha}} d x=\frac{1}{\alpha}\left(\frac{n}{2}\right)^{\alpha} \int_{0}^{\infty} \frac{\sin 2 x}{x^{\alpha}} d x \\
& =\frac{n^{\alpha}}{2 \alpha} \cos \frac{\pi \alpha}{2} \Gamma(1-\alpha)=\frac{n^{\alpha} \pi}{4 \sin (\pi \alpha / 2) \Gamma(\alpha+1)} .
\end{aligned}
$$


As before, we have to prove that

$$
\Delta=\int_{1-r}^{\infty} \frac{G(r, t)-G(t)}{t^{1+\alpha}} d t+\int_{0}^{1-r} \frac{G(r, t)}{t^{1+\alpha}} d t
$$

is bounded uniformly, if $G \in \operatorname{Lip} \alpha$. This is done again by observing that

$$
|G(r, t)-G(t)|=O(1-r)^{\alpha},
$$

and that

$$
G(r, t)=t\left(\frac{\partial G(r, t)}{\partial t}\right)_{t=\theta}=t O(1-r)^{\alpha-1} \quad(0<\theta<t) .
$$

Theorems III and IV concern Poisson's summability. If we assume more about the function $f$, we obtain corresponding theorems on equi-convergence of series and integrals.

TheOREM V. Let $f(x)=a_{0} / 2+\sum_{1}^{\infty}\left(a_{n} \cos n x+b_{n} \sin n x\right)$ be a continuous periodic function belonging to lip $\alpha(0<\alpha<1)$, of bounded variation, and of monotonic type. Then the difference

$$
\begin{aligned}
-\frac{1}{\pi} \Gamma(\alpha+1) \cos \frac{\pi \alpha}{2} \int_{1 / n}^{\infty} \frac{f(x+t)-f(x-t)}{t^{1+\alpha}} d t \\
\quad-\sum_{1}^{n}\left(a_{k} \sin k x-b_{k} \cos k x\right) k^{\alpha}
\end{aligned}
$$

tends to zero uniformly in $x$ as $n \rightarrow \infty$.

If $f \in \operatorname{Lip} \alpha$, the difference is uniformly bounded.

We base our proof on the following two lemmas whose proof appears elsewhere (see Salem and Zygmund [7]).

Lemma 1. If the continuous function $f(x)$ of period $2 \pi$ belongs to $\operatorname{Lip} \alpha$ $(0<\alpha<1)$ and is of monotonic type, then

$$
\left|S_{n}(x)-f(x)\right|=O\left(n^{-\alpha}\right),
$$

$s_{n}(x)$ being the partial sum of order $n$ of the Fourier series of $f(x)$. If $f \in \operatorname{lip} \alpha$, then $s_{n}-f=o\left(n^{-\alpha}\right)$.

Lemma 2. If $s_{n}(x)$ is the partial sum of order $n$ of the Fourier series of $f(x)$ and if $s_{n}(x)-f(x)=O\left(n^{-\alpha}\right), 0<\alpha<1$, uniformly in $x$, then the derivative $s_{n}^{\prime}(x)$ is $O\left(n^{1-\alpha}\right)$ uniformly in $x$. If $s_{n}-f=o\left(n^{-\alpha}\right)$, then $s_{n}^{\prime}=o\left(n^{1-\alpha}\right)$.

We can now prove our theorem. As in Theorem III, let

$$
g(t)=f(x+t)-f(x-t)=-\sum_{1}^{\infty} 2 \sin n t\left[a_{n} \sin n x-b_{n} \cos n x\right],
$$


and let

$$
s_{n}(t)=-\sum_{1}^{n} 2 \sin k t\left[a_{k} \sin k x-b_{k} \cos k x\right] .
$$

We have, as in Theorem III,

$$
-\frac{1}{\pi} \cos \frac{\pi \alpha}{2} \Gamma(\alpha+1) \int_{0}^{\infty} \frac{s_{n}(t)}{t^{1+\alpha}} d t=\sum_{1}^{n}\left(a_{k} \sin k x-b_{k} \cos k x\right) k^{\alpha}
$$

and thus we have to prove that

$$
D=\int_{0}^{\infty} \frac{s_{n}(t)}{t^{1+\alpha}} d t-\int_{1 / n}^{\infty} \frac{g(t)}{t^{1+\alpha}} d t
$$

is $O(1)$ if $f \in \operatorname{Lip} \alpha$, and $o(1)$ if $f \in \operatorname{lip} \alpha$. The proof being the same in both cases, we assume that $f \in \operatorname{Lip} \alpha$.

Write

$$
D=\int_{1 / n}^{\infty} \frac{s_{n}(t)-g(t)}{t^{1+\alpha}} d t+\int_{0}^{1 / n} \frac{s_{n}(t)}{t^{1+\alpha}} d t
$$

By Lemma $1, s_{n}-g$ is $O\left(n^{-\alpha}\right)$ uniformly in $x$ and $t$, and thus the first integral is

$$
O\left(n^{-\alpha} \int_{1 / n}^{\infty} \frac{d t}{t^{1+\alpha}}\right)=O(1)
$$

For the second integral, we note that $s_{n}(t)=s_{n}(t)-s_{n}(0)=t s_{n}^{\prime}(\theta), 0<\theta<t$, and thus, by Lemma $2, s_{n}(t)=O\left(t n^{1-\alpha}\right)$. Hence the second integral is

$$
O\left[n^{1-\alpha} \int_{0}^{1 / n} \frac{d t}{t^{\alpha}}\right]=O(1)
$$

which completes the proof of the theorem.

Theorem IV has also its corresponding equi-convergence theorem, under the same assumptions as stated in Theorem V.

THEOREM VI. The function $f(x)$ being of period $2 \pi$, continuous, of bounded variation, and of monotonic type, the difference

$$
\begin{array}{r}
-\frac{1}{\pi} \sin \frac{\pi \alpha}{2} \Gamma(\alpha+1) \int_{1 / n}^{\infty} \frac{f(x+t)+f(x-t)-2 f(x)}{t^{1+\alpha}} d t \\
-\sum_{1}^{n}\left(a_{k} \cos k x+b_{k} \sin k x\right) k^{\alpha}
\end{array}
$$

tends to zero uniformly in $x$ as $n \rightarrow \infty$ if $f \in \operatorname{lip} \alpha$, and is uniformly bounded if $f \in \operatorname{Lip} \alpha$.

The argument is essentially the same as in Theorem V, and we need only sketch it. Writing 


$$
\begin{gathered}
G(t)=f(x+t)+f(x-t)-2 f(x)=-4 \sum_{1}^{\infty} \sin ^{2} \frac{k t}{2}\left(a_{k} \cos k x+b_{k} \sin k x\right), \\
s_{n}(t)=-4 \sum_{1}^{n} \sin ^{2} \frac{k t}{2}\left(a_{k} \cos k x+b_{k} \sin k x\right),
\end{gathered}
$$

we have, as in Theorem IV,

$$
-\frac{1}{\pi} \sin \frac{\pi \alpha}{2} \Gamma(\alpha+1) \int_{0}^{\infty} \frac{s_{n}(t)}{t^{1+\alpha}} d t=\sum_{1}^{n}\left(a_{k} \cos k x+b_{k} \sin k x\right) k^{\alpha}
$$

and thus it remains to prove that

$$
\Delta=\int_{0}^{\infty} \frac{s_{n}(t)}{t^{1+\alpha}} d t-\int_{1 / n}^{\infty} \frac{G(t)}{t^{1+\alpha}} d t=\int_{1 / n}^{\infty} \frac{s_{n}(t)-G(t)}{t^{1+\alpha}} d t+\int_{0}^{1 / n} \frac{s_{n}(t)}{t^{1+\alpha}} d t
$$

is uniformly bounded if $f \in \operatorname{Lip} \alpha$. The proof, based on the two lemmas formulated above, is the same as in Theorem V.

Corollary. Suppose that $d f \sim a_{0} / 2+\sum\left(a_{n} \cos n x+b_{n} \sin n x\right)$ is the Fourier-Stieltjes series of a nondecreasing function, such that the Fourier series $\sum_{1}^{\infty}\left(a_{n} \cos n x+b_{n} \sin n x\right) / n^{1-\alpha}$ represents a bounded function $(0<\alpha<1)$. Then the partial sums of both series

$$
\sum_{1}^{\infty} \frac{a_{n} \cos n x+b_{n} \sin n x}{n^{1-\alpha}}
$$

$$
\sum_{1}^{\infty} \frac{a_{n} \sin n x-b_{n} \cos n x}{n^{1-\alpha}}
$$

are uniformly bounded.

In fact, the argument of Theorem I shows immediately that our hypothesis implies the boundedness of both functions $\mathrm{S}$ and $\mathrm{T}$ and by the argument of the Corollary of Theorem I we see that both functions

$$
\sum_{1}^{\infty} \frac{a_{n} \sin n x-b_{n} \cos n x}{n}=f-\frac{a_{0}}{2} x \text { and } \sum_{1}^{\infty} \frac{a_{n} \cos n x+b_{n} \sin n x}{n}
$$

belong to Lip $\alpha$. Hence, Theorems III and IV prove that the corresponding integrals are uniformly bounded. Then, applying Theorems V and VI, we see that both series $\mathrm{S}$ and $\mathrm{T}$ have their partial sums uniformly bounded.

In particular, by Theorem I, our corollary is true if $d f$ is a positive distribution concentrated over a set $E$ of positive $\alpha$-capacity, such that its potential with respect to $1 / r^{\alpha}$ is bounded.

\section{Theorems on the existence of certain integrals of the Dini type.}

THEOREM VII. Let $F(x)$ be continuous and nondecreasing in $(0,2 \pi)$ and belonging to $\operatorname{Lip} \alpha\left({ }^{2}\right)(0<\alpha<1)$. Then the points $x$ at which the integral

(2) As usual (see T.S. p. 8), we define $F(x)$ everywhere by the condition $F(x+2 \pi)-F(x)$ $=F(2 \pi)-F(0)$. 
$\int_{0}^{\pi}\left((F(x+t)-F(x-t)) / t^{1+\alpha}\right) d t$ is infinite form a set of $\alpha$-capacity zero.

The theorem holds for $\alpha=0$, the integral being in this case finite except in a set of logarithmic capacity zero.

Suppose first that $0<\alpha<1$. Let $d F \sim a_{0} / 2+\sum a_{n} \cos n x+b_{n} \sin n x$. By Theorem III applied to the periodic function $F(x)-\left(a_{0} / 2\right) x$, it is sufficient to prove that the points $x$ at which the function $F_{\alpha}(r, x)$ $=\sum\left(\left(a_{n} \cos n x+b_{n} \sin n x\right) / n^{1-\alpha}\right) r^{n}$ is unbounded as $r \rightarrow 1-0$ form a set of $\alpha$-capacity zero. This is an immediate consequence of our Theorem I and of a well known result in potential theory (see Frostman [2, pp. 81-82], de la Vallée Poussin $[3$, p. 21]) but the direct proof is so simple that we may give it here. Let $E$ be the set at which $F_{\alpha}(r, x)$ is unbounded (hence at which $\lim _{r \rightarrow 1} F_{\alpha}(r, x)$ $=+\infty)$, and suppose that the $\alpha$-capacity of $E$ is positive. Then by Theorem I there exists a positive distribution $d \mu \sim 1 / 2 \pi+\sum\left(\alpha_{n} \cos n x+\beta_{n} \sin n x\right)$ concentrated on $E$, such that $\mu_{\alpha}(r, x)=\sum\left(\left(\alpha_{n} \cos n x+\beta_{n} \sin n x\right) / n^{1-\alpha}\right) r^{n}$ is bounded as $r \rightarrow 1$. Henc , the integral $\int_{0}^{2 \pi} \mu_{\alpha}(r, x) d F$ is bounded as $r \rightarrow 1$. But this integral is equal to $\int_{0}^{2 \pi} F_{\alpha}(r, x) d \mu$ which tends to $+\infty$ as $r \rightarrow 1$ since the distribution $d \mu$ is concentrated on $E$ and $F_{\alpha}(r, x) \rightarrow+\infty$ in $E$. Hence the contradiction, which proves the, theorem.

For $\alpha=0$ the proof is identical, except that instead of our Theorem III we use the classical result (see T.S. p. 54)

$$
\frac{1}{\pi} \int_{1-r}^{\pi} \frac{F(x+t)-F(x-t)}{t} d t-\sum_{1}^{\infty} \frac{a_{n} \cos n x+b_{n} \sin n x}{n} r^{n}=o(1),
$$

Of course, $F(x)$ being nondecreasing, the existence of the integral $\int_{0}^{\pi}\left((F(x+t)-F(x-t)) / t^{1+\alpha}\right) d t$ implies the existence of the integrals

$$
\int_{0}^{\pi} \frac{F(x+t)-F(x)}{t^{1+\alpha}} d t, \quad \int_{0}^{\pi} \frac{F(x)-F(x-t)}{t^{1+\alpha}} d t,
$$

and so the existence of

$$
\int_{0}^{\pi} \frac{F(x+t)+F(x-t)-2 F(x)}{t^{1+\alpha}} d t
$$

as an absolutely convergent integral, outside a set of $\alpha$-capacity 0 .

It is a curious fact that each of the integrals:

$$
\begin{aligned}
& \int_{0}^{\infty} \frac{f(x+t)-f(x-t)}{t^{1+\alpha}} d t=\lim _{\leftrightarrow \rightarrow 0} \int_{e}^{\infty} \frac{f(x+t)-f(x-t)}{t^{1+\alpha}} d t, \\
& \begin{aligned}
\int_{0}^{\infty} \frac{f(x+t)+f(x-t)-2 f(x)}{t^{1+\alpha}} d t \\
=\lim _{\leftrightarrow \rightarrow 0} \int_{e}^{\infty} \frac{f(x+t)+f(x-t)-2 f(x)}{t^{1+\alpha}} d t
\end{aligned}
\end{aligned}
$$


may exist at some points even if we omit the condition that $f$ is nondecreasing.

Theorem VIII. Suppose that $f$ is of period $2 \pi$ and of the class lip $\alpha$ $(0<\alpha<1)$. Then the integral (1) exists in a set of points which is of the power of the continuum in every interval, though it may be of measure $0 . A$ similar result holds for the integral (2).

Proof. Without loss of generality we may assume that the constant term of the Fourier series of $f$ is 0 , so that

$$
f(x) \sim \sum_{n=1}^{\infty}\left(a_{n} \cos n x+b_{n} \sin n x\right) .
$$

We recall the following definition. A continuous function $F(x)$ is said to be $s m o o t h$, if

$$
\lim _{h \rightarrow 0} \frac{F(x+h)+F(x-h)-2 F(x)}{h}=0
$$

for each $x$. If (4) holds uniformly in $x$, the function $F$ will be called uniformly smooth.

It is known (see Zygmund [7]) that, if the function (3) is of the class $\operatorname{lip} \alpha, 0<\alpha<1$, its $(1-\alpha)$ integral

$$
\begin{aligned}
f_{1-\alpha}(x) \sim \sum_{n=1}^{\infty}\left\{a_{n} \cos \left[n x-(1-\alpha) \frac{\pi}{2}\right]\right. & \\
& \left.+b_{n} \sin \left[n x-(1-\alpha) \frac{\pi}{2}\right]\right\} n^{\alpha-1}
\end{aligned}
$$

is uniformly smooth. The same may be said of the function

$$
\begin{aligned}
\bar{f}_{1-\alpha}(x) \sim \sum_{n=1}^{\infty}\left\{a_{n} \sin \left[n x-(1-\alpha) \frac{\pi}{2}\right]\right. & \\
& \left.-b_{n} \cos \left[n x-(1-\alpha) \frac{\pi}{2}\right]\right\} n^{\alpha-1}
\end{aligned}
$$

since the function conjugate to a uniformly smooth function retains this property (loc. cit.).

It follows that the functions

$$
\begin{aligned}
& g(x) \sim \sum_{n=1}^{\infty}\left(a_{n} \cos n x+b_{n} \sin n x\right) n^{\alpha-1}, \\
& \bar{g}(x) \sim \sum_{n=1}^{\infty}\left(a_{n} \sin n x-b_{n} \cos n x\right) n^{\alpha-1}
\end{aligned}
$$

which are linear combinations of (5) and (6) are uniformly smooth. 
On the other hand it is also known (loc. cit.) that if $F(x)$ is smooth (it need not be uniformly smooth) it has a finite derivative in a set $E=E_{F}$ of points which is of the power of the continuum in every interval. Let $g(r, x)$ be the Poisson integral of $g(x)$. By the theorem of Fatou, $\lim _{r \rightarrow 1} \partial g(r, x) / \partial x$ exists at every point $x \in E_{g}$. In virtue of Theorem III, the integral (1) exists for $x \in E_{g}$. Similarly, using Theorem IV, we prove that (2) exists for $x \in E_{\bar{g}}$.

In order to show that the set of points at which, for example, the integral (1) converges may be of measure 0 , let us consider the function

$$
f(x)=\sum_{n=1}^{\infty} \delta_{n} 2^{-n \alpha} \sin 2^{n} x
$$

where, temporarily, $\left\{\delta_{n}\right\}$ is any sequence of numbers tending to 0 . It is easy to see that $f(x) \in \operatorname{lip} \alpha$ (see for example Zygmund [7]). Furthermore if $\epsilon=2^{-N}$

$$
\begin{aligned}
\int_{e}^{\infty} \frac{f(x+t)-f(x-t)}{t^{1+\alpha}} d t & =2 \sum_{n=1}^{\infty} \delta_{n} 2^{-n \alpha} \cos 2^{n} x \int_{\epsilon}^{\infty} \frac{\sin 2^{n} t}{t^{1+\alpha}} d t \\
& =\sum_{n=1}^{N}+\sum_{n=N+1}^{\infty}=A_{N}+B_{N}
\end{aligned}
$$

say. Obviously the terms of $B_{N}$ are $O\left(\delta_{n} 2^{-n \alpha} 2^{N \alpha}\right)$, so that

If $n \leqq N$, then

$$
B_{N}=o\left(2^{N \alpha} \sum_{n=N+1}^{\infty} 2^{-n \alpha}\right)=o(1)
$$

$$
2^{-n \alpha} \int_{2^{-N}}^{\infty} t^{-1-\alpha} \sin 2^{n} t d t=C-\int_{0}^{2^{n-N}} t^{-1-\alpha} \sin t d t=C-O\left(2^{(n-N)(1-\alpha)}\right)
$$

where $C=\int_{0}^{\infty} t^{-1-\alpha} \sin t d t$ is a constant different from 0 . Thus

$$
A_{N}=2 C \sum_{n=1}^{N} \delta_{n} \cos 2^{n} x+\sum_{n=1}^{N} o\left(2^{(n-N)(1-\alpha)}\right)=2 C \sum_{n=1}^{N} \delta_{n} \cos 2^{n} x+o(1) .
$$

It follows that, if $\epsilon=2^{-N}$, the left-hand side of (9) differs by $o$ (1) from the sum $2 C \sum_{1}^{N} \delta_{n} \cos 2^{n} x$. It is however well known that the latter sum is unbounded for almost every $x$ if $\sum \delta_{n}{ }^{2}=\infty$ (T.S. p. 120). Thus for the function (8) the integral (1) diverges almost everywhere if only $\delta_{n} \rightarrow 0, \sum \delta_{n}{ }^{2}=\infty$.

The same function $f$ will make integral (2) divergent almost everywhere.

Remarks. (i) The foregoing proof does not show whether (under the assumptions of Theorem VIII) there is at least one point at which (1) and (2) would exist simultaneously. The problem of the existence of such points remains open.

(ii) The conclusions of Theorem VIII remain valid if we assume only that $f(x+t)-f(x)=o\left(|t|^{\alpha}\right)$ as $t \rightarrow 0$, but not necessarily uniformly in $x$ (so that $f$ 
need not belong to lip $\alpha$ ). For under the new assumption the function $f_{1-\alpha}$ is still smooth (though not uniformly smooth), as shown in Zygmund [1]. The same argument also shows that $\bar{f}_{1-\alpha}$ is smooth. The rest of the argument is unchanged.

Determination of the $\alpha$-capacity of a symmetrical perfect set of the Cantor type. The results which we indicate in this last paragraph are not essentially new, but we think that the methods used to prove them present a certain interest.

Let the points of a symmetrical perfect set $P$ of the Cantor type constructed on $(0,2 \pi)$ be given by

$$
x=2 \pi\left[\epsilon_{1}\left(1-\xi_{1}\right)+\epsilon_{2} \xi_{1}\left(1-\xi_{2}\right)+\cdots+\epsilon_{k} \xi_{1} \cdots \xi_{k-1}\left(1-\xi_{k}\right)+\cdots\right],
$$

the $\xi_{k}\left(0<\xi_{k}<1 / 2\right)$ being the successive ratios of dissection of the intervals (in three parts proportional to $\xi_{k}, 1-2 \xi_{k}, \xi_{k}$ ) and the $\epsilon_{k}$ being 0 or 1. Let

$$
F(x)=\epsilon_{1} / 2+\epsilon_{2} / 2^{2}+\cdots+\epsilon_{k} / 2^{k}+\cdots
$$

be the values of the classical Lebesgue function constructed on $P$, when $x$ belongs to $P, F(x)$ being constant in each interval contiguous to $P . F(x)$ is continuous, monotonic nondecreasing.

It is easily seen (see, for example, Salem $\left[9\right.$, p. 24]) that, $\omega_{0}(\delta)$ being an increasing function of $\delta>0$ such that

$$
\omega_{0}\left(2 \pi \xi_{1} \cdots \xi_{k}\right)=4 \cdot 1 / 2^{k},
$$

$F(x)$ has a modulus of continuity not greater than $\omega_{0}(\delta)$.

We shall write

$$
r_{k}=2 \pi \xi_{1} \cdots \xi_{k-1}\left(1-\xi_{k}\right) .
$$

It is easily seen that the condition $\xi_{k}<1 / 2$ implies $r_{k}>r_{k+1}$.

Let us now suppose that the $\alpha$-capacity of $P$ is positive. Then the $\alpha$-Hausdorff measure of $P$ is also positive. This result is well known (see Frostman $[2$, p. 89]) but we can see it here as a consequence of our preceding theorems. For, by the corollary of Theorem I, there exists a positive distribution $\mu$ concentrated on $P$ and such that $\mu(x) \in \operatorname{Lip} \alpha$; and from this follows easily that the $\alpha$-Hausdorff measure of $P$ is positive. Hence we have

$$
\lim \inf 2^{k}\left(2 \pi \xi_{1} \cdots \xi_{k}\right)^{\alpha}=H>0
$$

as $k \rightarrow \infty$ and this, as we have said before, proves also that the modulus of continuity $\omega(\delta)$ of our function $F$ is $O\left(\delta^{\alpha}\right)$. Hence $F(x) \in \operatorname{Lip} \alpha$, if $\alpha$-capacity $(P)>0$.

Let now $x \in P$ and consider the integral $\int_{0}^{\pi}\left((F(x+t)-F(x-t)) / t^{1+\alpha}\right) d t$ (the function being extended outside $(0,2 \pi)$ as in Theorem VII). Suppose that $r_{k} \leqq t<r_{k-1}$ and observe that, for a given $x$ : 


$$
\begin{aligned}
\text { If } \epsilon_{k}=0, \text { then } F(x+t)-F(x-t) & >F(x+t)-F(x) \\
& \geqq F\left(x+r_{k}\right)-F(x)=1 / 2^{k} . \\
\text { If } \epsilon_{k}=1, \text { then } F(x+t)-F(x-t) & >F(x)-F(x-t) \\
& \geqq F(x)-F\left(x-r_{k}\right)=1 / 2^{k} .
\end{aligned}
$$

Hence in all cases

$$
\int_{r_{k}}^{r_{k-1}} \frac{F(x+t)-F(x-t)}{t^{1+\alpha}} d t>\frac{1}{2^{k}} \int_{r_{k}}^{r_{k-1}} \frac{d t}{t^{1+\alpha}}=\frac{1}{\alpha 2^{k}}\left[\frac{1}{r_{k}^{\alpha}}-\frac{1}{r_{k-1}^{\alpha}}\right] .
$$

Hence, by application of Theorems I and III, if the $\alpha$-capacity of $P$ is positive, the series $\sum_{k=2}^{\infty}\left[1 / r_{k}^{\alpha}-1 / r_{k-1}{ }^{\alpha}\right]\left(1 / 2^{k}\right)$ converges, and it is seen immediately that this implies the convergence of the series $\sum 1 / 2^{k} r_{k}{ }^{\alpha}$. Hence we have proved that if the set $P$ is of positive $\alpha$-capacity the series

is convergent.

$$
\sum_{1}^{\infty} \frac{1}{2^{k}\left(\xi_{1} \cdots \xi_{k}\right)^{\alpha}}
$$

Now we shall prove the converse, namely that the convergence of the last series implies that the set $P$ is of positive $\alpha$-capacity.

In fact, the convergence of the series implies first that

$$
\lim \inf 2^{k}\left(2 \pi \xi_{1} \cdots \xi_{k}\right)=H>0,
$$

so that the function $F(x)$ belongs to $\operatorname{Lip} \alpha$. If $\omega(\delta)$ denotes the modulus of continuity of $F(x)$, we have

$$
\sigma_{k}=\sum_{1 / r_{k-1} \leqq n<1 / r_{k}} \frac{1}{n^{1-\alpha}} \omega\left(\frac{1}{n}\right) \leqq \sum_{1 / r_{k-1} \leqq n<1 / r_{k}} \frac{1}{n^{1-\alpha}} \omega_{0}\left(\frac{1}{n}\right)
$$

where $\omega_{0}(\delta)$ is the above described function such that $\omega_{0}\left(r_{k}\right)=4 / 2^{k}$. Hence

$$
\sigma_{k}<A \frac{1}{2^{k}} \sum_{1 / r_{k-1} \leqq n<1 / r_{k}} \frac{1}{n^{1-\alpha}}=O\left(\frac{1}{2^{k} r_{k}^{\alpha}}\right) .
$$

Hence $\sum \sigma_{k}<\infty$, that is, $\sum_{1}^{\infty}\left(1 / n^{1-\alpha}\right) \omega(1 / n)<\infty$ : this implies the convergence of the integral $\int_{0}\left(\omega(t) / t^{1+\alpha}\right) d t$, that is to say the uniform convergence of $\int_{0}^{\pi}\left((F(x+t)-F(x-t)) / t^{1+\alpha}\right) d t$; and since $F \in \operatorname{Lip} \alpha$, the Theorems III and I prove that the set $P$ is of positive $\alpha$-capacity.

Hence the necessary and sufficient condition for the symmetrical perfect set $P$ of the Cantor type to be of positive $\alpha$-capacity is the convergence of the series $\sum_{1}^{\infty} 2^{-k}\left(\xi_{1} \cdots \xi_{k}\right)^{-\alpha}(0<\alpha<1)$. This result has already been obtained, in the particular case $\xi_{k}=$ const., by Pólya and Szegö, in a quite different way. See their paper on transfinite diameter (Pólya and Szegö [5]) and the result of von Neumann quoted there. 
This proves our former assertion that the existence of a positive distribution $\mu$ concentrated on a set $E$, and such that $\mu(x) \in \operatorname{Lip} \alpha$, is not sufficient in order that $\alpha$-capacity $(E)>0$. For take a set $P$ such that $2^{k}\left(\xi_{1} \cdots \xi_{k}\right)^{\alpha} \sim 1$ as $k \rightarrow \infty . F(x)$ is a distribution concentrated on $P$, and $F(x) \in \operatorname{Lip} \alpha$. But our series is divergent, and thus the $\alpha$-capacity of $P$ is zero.

The same argument as above would prove (even in a simpler way since here we need not investigate the modulus of continuity of $F$ ) that the necessary and sufficient condition for $P$ to be of positive logarithmic capacity is the convergence of the series $\sum_{1}^{\infty}\left(1 / 2^{k}\right) \log \left(1 / \xi_{k}\right)$. This last result is obtainable by different methods (see R. Nevanlinna [4, p. 149]).

Finally, we observe that our argument gives also the $\alpha$-capacity or the logarithmic capacity of symmetrical perfect sets of order $d$ (see Salem [9, pp. 23-24]). Such a set is of $\alpha$-positive capacity if and only if $\sum_{1}^{\infty}(d+1)^{-k}\left(\xi_{1} \cdots \xi_{k}\right)^{-\alpha}<\infty$, and of positive logarithmic capacity if and only if $\sum_{1}^{\infty}(d+1)^{-k} \log \left(1 / \xi_{k}\right)<\infty$. The proofs may be left to the reader.

\section{BIBLIOGRAPHY}

1. A. Beurling, Sur les ensembles exceptionnels, Acta Math. vol. 72 (1940).

2. O. Frostman, Potentiel d'équilibre et capacité des ensembles, Lund, 1935.

3. Ch. J. de la Vallee Poussin, Capacité des ensembles, Paris, 1937.

4. R. Nevanlinna, Eindeutige analytische Funktionen.

5. G. Polya and G. Szegö, Ueber den transfiniten Durchmesser, J. Reine Angew. Math. vol. 165 (1931).

6. R. Salem and A. Zygmund, The approximation by partial sums of Fourier series, Trans. Amer. Math. Soc. vol. 59 (1946).

7. A. Zygmund, Smooth functions, Duke Math. J. vol. 12 (1945).

8. - Trigonometrical series, Warsaw, 1935.

9. R. Salem, On a theorem of Zygmund, Duke Math. J. vol. 10 (1943).

Massachusetts Institute of Technology and HaRvard University, Cambridge, Mass.

Mount Holyoke College, South Hadley, Mass. 